Noname manuscript No.

(will be inserted by the editor)

\title{
From Wallis and Forsyth to Ramanujan
}

\author{
Paul Levrie • Amrik Singh Nimbran
}

the date of receipt and acceptance should be inserted later

Abstract We show how a couple of Ramanujan's series for $1 / \pi$ can be deduced directly from Forsyth's series and from Wallis's product formula for $\pi$. The same method is used to obtain Bauer's alternating series.

Mathematics Subject Classification (2000) 11Y60 - 33D15 - 05A10

Keywords Series for $1 / \pi$, Wallis's product formula, Ramanujan's series

\section{Introduction}

In 1883 the Cambridge mathematician A.R. Forsyth derived the following formula for $\frac{1}{\pi}$ using Legendre polynomials [5]:

$$
\sum_{n=0}^{\infty} \frac{\left(\begin{array}{c}
2 n \\
n
\end{array}\right)^{2}}{(2 n-1)^{2} 2^{4 n}}=\frac{4}{\pi}
$$

J.W.L. Glaisher [6] used elliptic functions to prove in 1905 a number of similar formulas including the following one:

$$
\sum_{n=0}^{\infty} \frac{\left(\begin{array}{c}
2 n \\
n
\end{array}\right)^{2}}{(n+1) 2^{4 n}}=\frac{4}{\pi}
$$

Paul Levrie

Faculty of Applied Engineering, University of Antwerp, Groenenborgerlaan 171, 2020 Antwerpen; Department of Computer Science, KU Leuven, P.O. Box 2402, B-3001 Heverlee, Belgium

E-mail: paul.levrie@cs.kuleuven.be

Amrik Singh Nimbran

B3-304, Palm Grove Heights, Ardee City, Gurgaon, Haryana, INDIA 122003

E-mail: amrikn622@gmail.com 
And there is also this series:

$$
-\sum_{n=0}^{\infty} \frac{\left(\begin{array}{c}
2 n \\
n
\end{array}\right)^{2}}{(2 n-1) 2^{4 n}}=\frac{2}{\pi} .
$$

Both (2) and (3) are equivalent with Euler's version of Wallis's famous product formula for $\pi$ derivable from the infinite product expansion of the sine function [14]:

$$
\begin{aligned}
\sum_{n=0}^{\infty} \frac{\left(\begin{array}{c}
2 n \\
n
\end{array}\right)^{2}}{(n+1) 2^{4 n}} & =1+\frac{1}{2 \cdot 2^{2}}+\frac{1^{2} 3^{2}}{2 \cdot 4^{2} 6}+\frac{1^{2} 3^{2} 5^{2}}{2 \cdot 4^{2} 6^{2} 8}+\frac{1^{2} 3^{2} 5^{2} 7^{2}}{2 \cdot 4^{2} 6^{2} 8^{2} 10}+\cdots \\
& =\frac{3 \cdot 3}{2 \cdot 4}+\frac{1^{2} 3^{2}}{2 \cdot 4^{2} 6}+\frac{1^{2} 3^{2} 5^{2}}{2 \cdot 4^{2} 6^{2} 8}+\frac{1^{2} 3^{2} 5^{2} 7^{2}}{2 \cdot 4^{2} 6^{2} 8^{2} 10}+\cdots \\
& =\frac{3 \cdot 3}{2 \cdot 4} \cdot \frac{5 \cdot 5}{4 \cdot 6}+\frac{1^{2} 3^{2} 5^{2}}{2 \cdot 4^{2} 6^{2} 8}+\frac{1^{2} 3^{2} 5^{2} 7^{2}}{2 \cdot 4^{2} 6^{2} 8^{2} 10}+\cdots \\
& =\frac{3 \cdot 3}{2 \cdot 4} \cdot \frac{5 \cdot 5}{4 \cdot 6} \cdot \frac{7 \cdot 7}{6 \cdot 8}+\frac{1^{2} 3^{2} 5^{2} 7^{2}}{2 \cdot 4^{2} 6^{2} 8^{2} 10}+\cdots \\
& =\frac{3 \cdot 3}{2 \cdot 4} \cdot \frac{5 \cdot 5}{4 \cdot 6} \cdot \frac{7 \cdot 7}{6 \cdot 8} \cdots=\frac{4}{\pi}
\end{aligned}
$$

and

$$
\begin{aligned}
-\sum_{n=0}^{\infty} \frac{\left(\begin{array}{c}
2 n \\
n
\end{array}\right)^{2}}{(2 n-1) 2^{4 n}} & =1-\frac{1}{2^{2}}-\frac{1^{2} 3}{2^{2} 4^{2}}-\frac{1^{2} 3^{2} 5}{2^{2} 4^{2} 6^{2}}-\frac{1^{2} 3^{2} 5^{2} 7}{2^{2} 4^{2} 6^{2} 8^{2}}-\cdots \\
& =\frac{1 \cdot 3}{2 \cdot 2}-\frac{1^{2} 3}{2^{2} 4^{2}}-\frac{1^{2} 3^{2} 5}{2^{2} 4^{2} 6^{2}}-\frac{1^{2} 3^{2} 5^{2} 7}{2^{2} 4^{2} 6^{2} 8^{2}}-\cdots \\
& =\frac{1 \cdot 3}{2 \cdot 2} \cdot \frac{3 \cdot 5}{4 \cdot 4}-\frac{1^{2} 3^{2} 5}{2^{2} 4^{2} 6^{2}}-\frac{1^{2} 3^{2} 5^{2} 7}{2^{2} 4^{2} 6^{2} 8^{2}}-\cdots \\
& =\frac{1 \cdot 3}{2 \cdot 2} \cdot \frac{3 \cdot 5}{4 \cdot 4} \cdot \frac{5 \cdot 7}{6 \cdot 6}-\frac{1^{2} 3^{2} 5^{2} 7}{2^{2} 4^{2} 6^{2} 8^{2}}-\cdots \\
& =\frac{1 \cdot 3}{2 \cdot 2} \cdot \frac{3 \cdot 5}{4 \cdot 4} \cdot \frac{5 \cdot 7}{6 \cdot 6} \cdots=\frac{2}{\pi} .
\end{aligned}
$$

The following series is due to G. Bauer (1859) [10]:

$$
\sum_{n=0}^{\infty}(-1)^{n} \frac{(4 n+1)\left(\begin{array}{c}
2 n \\
n
\end{array}\right)^{3}}{2^{6 n}}=\frac{2}{\pi} .
$$

All the previous series converge very slowly. The last one can be found in S. Ramanujan's first letter to G. Hardy, dated January 31, 1913. In a paper published in 1914 [16] Ramanujan lists some more rapidly converging series for $1 / \pi$ which he found using modular equations. These are two of them:

$$
\sum_{n=0}^{\infty} \frac{(6 n+1)\left(\begin{array}{c}
2 n \\
n
\end{array}\right)^{3}}{2^{8 n}}=\frac{4}{\pi},
$$




$$
\sum_{n=0}^{\infty} \frac{(42 n+5)\left(\begin{array}{c}
2 n \\
n
\end{array}\right)^{3}}{2^{12 n}}=\frac{16}{\pi}
$$

In this paper we show how Ramanujan's series (5) and a number of other series for $\frac{1}{\pi}$ can be derived from Forsyth's in a straightforward way. The same method can also be applied to Glaisher's series (2) and to (3), leading to a proof of (4) and (6).

Series for $\frac{1}{\pi}$ have received much attention lately, see for instance the survey paper by Baruah, Berndt and Chan [2] and the work of Guillera [7,8] who uses the Wilf-Zeilberger method to find series of this form. Chu [3] and Liu [11, 12] have obtained similar results by manipulating hypergeometric series using Dougall's and Gauss's summation formulae.

\section{A first recurrence}

Note that all the series in the introduction contain the central binomial coefficients which can be rewritten using the Pochhammer symbol:

$$
\left(\begin{array}{c}
2 n \\
n
\end{array}\right)=2^{2 n} \frac{\left(\frac{1}{2}\right)_{n}}{n !}=2^{2 n} \frac{\left(\frac{1}{2}\right)_{n}}{(1)_{n}} .
$$

Using this in the general term of (1) we find that:

$$
\frac{\left(\begin{array}{c}
2 n \\
n
\end{array}\right)^{2}}{(2 n-1)^{2} 2^{4 n}}=\frac{1}{(2 n-1)^{2}} \frac{\left(\frac{1}{2}\right)_{n}^{2}}{(1)_{n}^{2}}=\frac{1}{4} \frac{\left(\frac{1}{2}\right)_{n-1}^{2}}{(1)_{n}^{2}}=\frac{1}{4} \frac{\left(\frac{1}{2}\right)_{n-1}^{2}}{(2)_{n-1}^{2}}
$$

Hence Forsyth's series can be written in the following form:

$$
\frac{4}{\pi}=1+\frac{1}{4} \sum_{n=0}^{\infty} \frac{\left(\frac{1}{2}\right)_{n}^{2}}{(2)_{n}^{2}}
$$

This last series is a special case of the more general series

$$
\sum_{n=0}^{\infty} \frac{(a)_{n}^{2}}{(a+b)_{n}^{2}}={ }_{3} F_{2}\left[\begin{array}{ccc}
a & a & 1 \\
a+b & a+b
\end{array} ; 1\right]
$$

with $a=\frac{1}{2}$ and $b=\frac{3}{2}$. In the rest of the paper we will use the abbreviation:

$$
s(a, b)=\sum_{n=0}^{\infty} \frac{(a)_{n}^{2}}{(a+b)_{n}^{2}} .
$$

This expression satisfies the following recurrence relation:

Theorem $1(+0,+1-$ scheme $)$

$$
s(a, b)=\frac{2 a+3 b-2}{2(2 b-1)}+\frac{b^{3}}{2(2 b-1)(a+b)^{2}} \cdot s(a, b+1) .
$$


Proof Manipulation of the general term in the series $s(a, b+1)$ leads to the required result. We start by writing:

$$
\sum_{n=0}^{\infty} \frac{(a)_{n}^{2}}{(a+b+1)_{n}^{2}}=\frac{1}{b^{2}} \sum_{n=0}^{\infty} \frac{(a)_{n}^{2}(a+b+n-(a+n))^{2}}{(a+b+1)_{n}^{2}} .
$$

If we work out the numerator, we get the following 3 series:

$$
\begin{aligned}
\sum_{n=0}^{\infty} \frac{(a)_{n}^{2}(a+b+n)^{2}}{(a+b+1)_{n}^{2}} & =(a+b)^{2} \sum_{n=0}^{\infty} \frac{(a)_{n}^{2}}{(a+b)_{n}^{2}}=(a+b)^{2} s(a, b) \\
\sum_{n=0}^{\infty} \frac{(a)_{n}^{2}(a+n)^{2}}{(a+b+1)_{n}^{2}} & =(a+b)^{2} \sum_{n=0}^{\infty} \frac{(a)_{n+1}^{2}}{(a+b)_{n+1}^{2}}=(a+b)^{2}(s(a, b)-1) \\
\sum_{n=0}^{\infty} \frac{(a)_{n}^{2}(a+b+n)(a+n)}{(a+b+1)_{n}^{2}} & =(a+b)^{2} \sum_{n=0}^{\infty} \frac{(a)_{n}^{2}(a+n)}{(a+b)_{n}^{2}(a+b+n)} .
\end{aligned}
$$

We now deal with this last series. Using a similar trick as before, we get:

$$
\begin{aligned}
\sum_{n=0}^{\infty} \frac{(a)_{n}^{2}(a+n)}{(a+b)_{n}^{2}(a+b+n)} & =\frac{1}{b} \sum_{n=0}^{\infty} \frac{(a)_{n}^{2}(a+n)(a+b+n-(a+n))}{(a+b)_{n}^{2}(a+b+n)} \\
& =\frac{1}{b}\left(\sum_{n=0}^{\infty} \frac{(a)_{n}^{2}(a+n)}{(a+b)_{n}^{2}}-\sum_{n=0}^{\infty} \frac{(a)_{n+1}^{2}}{(a+b)_{n}^{2}(a+b+n)}\right) \\
& =\frac{1}{b}\left(a+\sum_{n=1}^{\infty} \frac{(a)_{n}^{2}(a+n)}{(a+b)_{n}^{2}}-\sum_{n=0}^{\infty} \frac{(a)_{n+1}^{2}}{(a+b)_{n}^{2}(a+b+n)}\right) \\
& =\frac{1}{b}\left(a+\sum_{n=0}^{\infty} \frac{(a)_{n+1}^{2}(a+n+1)}{(a+b)_{n+1}^{2}}-\sum_{n=0}^{\infty} \frac{(a)_{n+1}^{2}}{(a+b)_{n}^{2}(a+b+n)}\right) \\
& =\frac{a}{b}+\frac{1}{b} \sum_{n=0}^{\infty} \frac{(a)_{n+1}^{2}(a+n+1-(a+b+n))}{(a+b)_{n+1}^{2}} \\
& =\frac{a}{b}+\frac{1-b}{b} \sum_{n=0}^{\infty} \frac{(a)_{n+1}^{2}}{(a+b)_{n+1}^{2}}=\frac{a}{b}+\frac{1-b}{b}(s(a, b)-1) .
\end{aligned}
$$

We bring everything together:

$$
s(a, b+1)=\frac{(a+b)^{2}}{b^{2}}\left(s(a, b)+s(a, b)-1-2\left(\frac{a}{b}+\frac{1-b}{b}(s(a, b)-1)\right)\right) .
$$

Rearranging leads to (9).

Note that this theorem can be found in a slightly less general form in Knopp [9, p. 261-262]. It is a special case of Kummer's transformation of series.

Using Theorem 1 we are now able to rewrite $s(a, b)$ : 
Theorem 2

$$
s(a, b)=\sum_{n=0}^{\infty} \frac{2 a+3(b+n)-2}{2(2(b+n)-1)} \cdot \frac{(b)_{n}^{3}}{2^{2 n}\left(b-\frac{1}{2}\right)_{n}(a+b)_{n}^{2}} .
$$

Proof From (9) it follows that:

$s(a, b+n)=\frac{2 a+3(b+n)-2}{2(2(b+n)-1)}+\frac{(b+n)^{3}}{2^{2}\left(b+n-\frac{1}{2}\right)(a+b+n)^{2}} \cdot s(a, b+n+1)$.

Iterating this formula starting from $n=0$ proves the result.

Ramanujan's formula (5) is an immediate consequence of Theorem 2:

Corollary 1

$$
\sum_{n=0}^{\infty} \frac{(6 n+1)\left(\begin{array}{c}
2 n \\
n
\end{array}\right)^{3}}{2^{8 n}}=\frac{4}{\pi}
$$

Proof Forsyth's formula is equivalent with (7):

$$
\frac{16}{\pi}-4=s\left(\frac{1}{2}, \frac{3}{2}\right) \text {. }
$$

We rewrite the right-hand side using Theorem 2:

$$
\begin{aligned}
s\left(\frac{1}{2}, \frac{3}{2}\right) & =\sum_{n=0}^{\infty} \frac{2+3(3+2 n)-4}{2(2(3+2 n)-2)} \cdot \frac{\left(\frac{3}{2}\right)_{n}^{3}}{2^{2 n}(1)_{n}(2)_{n}^{2}} \\
& =\sum_{n=0}^{\infty} \frac{6 n+7}{8} \cdot \frac{8\left(\frac{1}{2}\right)_{n+1}^{3}}{2^{2 n}(1)_{n+1}^{3}} \\
& =4 \sum_{n=1}^{\infty} \frac{(6 n+1)\left(\begin{array}{c}
2 n \\
n
\end{array}\right)^{3}}{2^{8 n}} .
\end{aligned}
$$

This leads to:

$$
\frac{16}{\pi}=4+4 \sum_{n=1}^{\infty} \frac{(6 n+1)\left(\begin{array}{c}
2 n \\
n
\end{array}\right)^{3}}{2^{8 n}} \Rightarrow \frac{4}{\pi}=\sum_{n=0}^{\infty} \frac{(6 n+1)\left(\begin{array}{c}
2 n \\
n
\end{array}\right)^{3}}{2^{8 n}}
$$

\section{Other recurrences}

Note that the following recurrence relation:

$$
s(a, b)=1+\frac{a^{2}}{(a+b)^{2}} s(a+1, b)
$$

is related to the series (8) in the same way that (9) is related to (10). By combining (9) and (11) we get new recurrences. 
Theorem $3(+1,+1-$ scheme)

$s(a, b)=\frac{(2 a+3 b-2)(a+b)^{2}+b^{3}}{2(2 b-1)(a+b)^{2}}+\frac{a^{2} b^{3}}{2(2 b-1)(a+b)^{2}(a+b+1)^{2}} s(a+1, b+1)$.

The corresponding series is:

$s(a, b)=\sum_{n=0}^{\infty} \frac{(5 n+2 a+3 b-2)(a+b+2 n)^{2}+(b+n)^{3}}{2(2(b+n)-1)(a+b+2 n)^{2}} \cdot \frac{(a)_{n}^{2}(b)_{n}^{3}}{2^{2 n}\left(b-\frac{1}{2}\right)_{n}(a+b)_{2 n}^{2}}$.

Proof We replace $s(a, b+1)$ in the right-hand side of (9) by the corresponding formula from (11):

$$
\begin{aligned}
s(a, b) & =\frac{2 a+3 b-2}{2(2 b-1)}+\frac{b^{3}}{2(2 b-1)(a+b)^{2}} \cdot s(a, b+1) \\
& =\frac{2 a+3 b-2}{2(2 b-1)}+\frac{b^{3}}{2(2 b-1)(a+b)^{2}} \cdot\left(1+\frac{a^{2}}{(a+b+1)^{2}} s(a+1, b+1)\right) .
\end{aligned}
$$

The series follows immediately from this recurrence.

If we take $a=\frac{1}{2}, b=\frac{3}{2}$, we get the following series:

$$
\frac{4}{\pi}=\sum_{n=0}^{\infty} \frac{168 n^{3}-36 n^{2}+6 n+1}{(2 n-1)^{2}} \frac{\left(\begin{array}{c}
2 n \\
n
\end{array}\right)^{3}}{2^{12 n}} .
$$

By combining the two recurrences (9) and (11) we can derive other series. For instance, if we use (11) again in the recurrence of Theorem 3, we get this series $(+2,+1$-scheme):

$$
\frac{4}{\pi}=\sum_{n=0}^{\infty} \frac{18560 n^{5}-20736 n^{4}+8160 n^{3}-992 n^{2}+30 n+9}{(4 n-1)^{2}(4 n-3)^{2}} \frac{\left(\begin{array}{c}
2 n \\
n
\end{array}\right)\left(\begin{array}{c}
4 n \\
n
\end{array}\right)^{2}}{2^{16 n}} .
$$

And this is the series we obtain if we use (9) again in the recurrence of Theorem $3(+1,+2-$ scheme $)$ :

$$
\frac{4}{\pi}=\sum_{n=0}^{\infty} \frac{186368 n^{5}-128000 n^{4}+22304 n^{3}-152 n^{2}+2 n+1}{(2 n-1)(4 n-1)^{3}} \frac{\left(\begin{array}{c}
4 n \\
2 n
\end{array}\right)\left(\begin{array}{c}
4 n \\
n
\end{array}\right)^{2}}{2^{20}} .
$$

\section{Recurrences related to Glaisher's and Wallis's series}

Glaisher's series (2) can be written in the following form:

$$
\frac{4}{\pi}=1+\frac{1}{4} \sum_{n=0}^{\infty} \frac{\left(\frac{3}{2}\right)_{n}^{2}}{(2)_{n}(2)_{n+1}}
$$


The series on the right-hand side is the special case $a=\frac{3}{2}, b=\frac{1}{2}$ of this series:

$$
t(a, b)=\sum_{n=0}^{\infty} \frac{(a)_{n}^{2}}{(a+b)_{n}(a+b)_{n+1}} .
$$

In a similar way as in the previous sections we can prove the two recurrences equivalent with (9) and (11) for $t$ :

$$
\begin{gathered}
t(a, b)=\frac{1}{a+b}+\frac{a^{2}}{(a+b)^{2}} t(a+1, b), \\
t(a, b)=\frac{a+2 a b+b+3 b^{2}}{2(2 b+1) b(a+b)}+\frac{b(b+1)^{2}}{2(2 b+1)(a+b)^{2}} t(a, b+1) .
\end{gathered}
$$

Using only the last recurrence $\left(+0,+1\right.$-scheme) with $a=\frac{3}{2}$ and $b=\frac{1}{2}$ we get the following new series:

$$
\frac{4}{\pi}=\sum_{n=0}^{\infty} \frac{12 n^{2}+4 n+1}{(2 n-1)^{2}(n+1)} \frac{\left(\begin{array}{c}
2 n \\
n
\end{array}\right)^{3}}{2^{8 n}} .
$$

With the $+1,+1$-scheme we again obtain the series (12).

The series (3) can be rewritten like this:

$$
\frac{2}{\pi}=1-\frac{1}{8} \sum_{n=0}^{\infty} \frac{\left(\frac{3}{2}\right)_{n-1}\left(\frac{3}{2}\right)_{n}}{(2)_{n}^{2}} .
$$

where the series on the right is a special case of

$$
u(a, b)=\sum_{n=0}^{\infty} \frac{(a)_{n-1}(a)_{n}}{(a+b)_{n}^{2}}
$$

with corresponding recurrences:

$$
\begin{gathered}
u(a, b)=\frac{1}{a-1}+\frac{a^{2}}{(a+b)^{2}} u(a+1, b), \\
u(a, b)=\frac{a+2 a b+3 b^{2}-1}{2(2 b+1) b(a-1)}+\frac{b(b+1)^{2}}{2(2 b+1)(a+b)^{2}} u(a, b+1) .
\end{gathered}
$$

The $+0,+1-$ scheme leads in this case to the series:

$$
\frac{2}{\pi}=-\sum_{n=0}^{\infty} \frac{12 n^{2}-1}{(2 n-1)^{2}} \frac{\left(\begin{array}{c}
2 n \\
n
\end{array}\right)^{3}}{2^{8 n}} .
$$

With the $+1,+1$-scheme we find this series:

$$
\frac{2}{\pi}=-\sum_{n=0}^{\infty} \frac{168 n^{3}+20 n^{2}-2 n-1}{(2 n-1)^{2}} \frac{\left(\begin{array}{c}
2 n \\
n
\end{array}\right)^{3}}{2^{12 n}} .
$$

With these series we can prove (6): 


\section{Corollary 2}

$$
\sum_{n=0}^{\infty} \frac{(42 n+5)\left(\begin{array}{c}
2 n \\
n
\end{array}\right)^{3}}{2^{12 n}}=\frac{16}{\pi}
$$

Proof If we add (12) and (13), we get a new series:

$$
\frac{3}{\pi}=\sum_{n=0}^{\infty} \frac{-28 n^{2}+4 n+1}{(2 n-1)^{2}} \frac{\left(\begin{array}{c}
2 n \\
n
\end{array}\right)^{3}}{2^{12 n}} .
$$

If we substract (13) from 6 times the previous series, the sum of the new series is $\frac{16}{\pi}$, and the polynomial in the numerator is given by:

$$
6\left(-28 n^{2}+4 n+1\right)+168 n^{3}+20 n^{2}-2 n-1=(42 n+5)(2 n-1)^{2} .
$$

Hence the resulting series is Ramanujan's series (6).

Note that (3) can also be written in this form:

$$
\frac{2}{\pi}=1-\frac{1}{4} \sum_{n=0}^{\infty} \frac{\left(\frac{1}{2}\right)_{n}\left(\frac{3}{2}\right)_{n}}{(2)_{n}^{2}}
$$

and hence is a special case $\left(a=\frac{1}{2}, b=\frac{3}{2}\right)$ of this series:

$$
v(a, b)=\sum_{n=0}^{\infty} \frac{(a)_{n}(b)_{n}}{(a+b)_{n}^{2}}={ }_{3} F_{2}\left[\begin{array}{ccc}
a & b & 1 \\
a+b & a+b
\end{array} ; 1\right] .
$$

The corresponding recurrences are:

\section{Theorem 4}

$$
\begin{aligned}
& v(a, b)=\frac{a+2 b-1}{a+b-1}-\frac{b^{3}}{(a+b)^{2}(a+b-1)} \cdot v(a, b+1), \\
& v(a, b)=\frac{2 a+b-1}{a+b-1}-\frac{a^{3}}{(a+b)^{2}(a+b-1)} \cdot v(a+1, b) .
\end{aligned}
$$

Proof We prove the first one. The second one follows by symmetry. Note that the identity we want to prove can be rewritten like this:

$$
v(a, b+1)=\frac{(a+b)^{2}}{b^{2}}\left(1-\frac{a+b-1}{b}(v(a, b)-1)\right)
$$

or

$$
\sum_{n=0}^{\infty} \frac{(a)_{n}(b+1)_{n}}{(a+b+1)_{n}^{2}}=\frac{(a+b)^{2}}{b^{2}}\left(1-\frac{a+b-1}{b}(v(a, b)-1)\right) .
$$

We use the definition of the pochhammer symbol to rewrite the left-hand side and at the same time we add a factor:

$$
\sum_{n=0}^{\infty} \frac{(a)_{n}(b+1)_{n}}{(a+b+1)_{n}^{2}}=\frac{(a+b)^{2}}{b^{2}} \sum_{n=0}^{\infty} \frac{(a)_{n}(b)_{n+1}}{(a+b)_{n+1}^{2}}(a+b+n-(a+n)) .
$$


After simplifying and using the definition of $v$, what we have to prove becomes:

$\sum_{n=0}^{\infty} \frac{(a)_{n}(b)_{n+1}}{(a+b)_{n+1}^{2}}(a+b+n)-\sum_{n=0}^{\infty} \frac{(a)_{n+1}(b)_{n+1}}{(a+b)_{n+1}^{2}}=1-\frac{a+b-1}{b} \sum_{n=0}^{\infty} \frac{(a)_{n+1}(b)_{n+1}}{(a+b)_{n+1}^{2}}$.

Note that two sums cancel out, and we are left with:

$$
\sum_{n=0}^{\infty} \frac{(a)_{n}(b)_{n+1}}{(a+b)_{n+1}^{2}}(a+b+n)=1-\frac{a-1}{b} \sum_{n=0}^{\infty} \frac{(a)_{n+1}(b)_{n+1}}{(a+b)_{n+1}^{2}}
$$

We multiply by $b$ and rewrite the left-hand side:

$$
\begin{aligned}
& \sum_{n=0}^{\infty} \frac{(a)_{n}(b)_{n+1}}{(a+b)_{n+1}^{2}}(a+b+n)(a+b+n-(a+n))=b-(a-1) \sum_{n=0}^{\infty} \frac{(a)_{n+1}(b)_{n+1}}{(a+b)_{n+1}^{2}} \\
& \Leftrightarrow \sum_{n=0}^{\infty} \frac{(a)_{n}(b)_{n+1}}{(a+b)_{n}^{2}}-\sum_{n=0}^{\infty} \frac{(a)_{n+1}(b)_{n+1}}{(a+b)_{n+1}^{2}}(a+b+n) \\
& =b-(a-1) \sum_{n=0}^{\infty} \frac{(a)_{n+1}(b)_{n+1}}{(a+b)_{n+1}^{2}} \\
& \Leftrightarrow \sum_{n=0}^{\infty} \frac{(a)_{n}(b)_{n+1}}{(a+b)_{n}^{2}}-\sum_{n=0}^{\infty} \frac{(a)_{n+1}(b)_{n+1}}{(a+b)_{n+1}^{2}}(b+n)=b+\sum_{n=0}^{\infty} \frac{(a)_{n+1}(b)_{n+1}}{(a+b)_{n+1}^{2}} .
\end{aligned}
$$

If we change the index of summation in the first term on the left, the $b$ at the right cancels out:

$$
\sum_{n=0}^{\infty} \frac{(a)_{n+1}(b)_{n+1}(b+n+1)}{(a+b)_{n+1}^{2}}-\sum_{n=0}^{\infty} \frac{(a)_{n+1}(b)_{n+1}(b+n)}{(a+b)_{n+1}^{2}}=\sum_{n=0}^{\infty} \frac{(a)_{n+1}(b)_{n+1}}{(a+b)_{n+1}^{2}} .
$$

It is now easy to see that both sides are equal.

Using the first recurrence in the same way as in Theorem 2, we get the following result:

\section{Theorem 5}

$$
v(a, b)=\sum_{n=0}^{\infty}(-1)^{n} \frac{a+2(b+n)-1}{a+b+n-1} \frac{(b)_{n}^{3}}{(a+b)_{n}^{2}(a+b-1)_{n}} .
$$

An immediate consequence is (4):

\section{Corollary 3}

$$
\sum_{n=0}^{\infty}(-1)^{n} \frac{(4 n+1)\left(\begin{array}{c}
2 n \\
n
\end{array}\right)^{3}}{2^{6 n}}=\frac{2}{\pi}
$$


Proof We rewrite the right-hand side of (14) using Theorem 5:

$$
\begin{aligned}
v\left(\frac{1}{2}, \frac{3}{2}\right) & =\sum_{n=0}^{\infty}(-1)^{n} \frac{1+2(3+2 n)-2}{2 n+2} \cdot \frac{\left(\frac{3}{2}\right)_{n}^{3}}{(1)_{n}(2)_{n}^{2}} \\
& =\sum_{n=0}^{\infty}(-1)^{n} \frac{4 n+5}{2} \cdot \frac{8\left(\frac{1}{2}\right)_{n+1}^{3}}{(1)_{n+1}^{3}} \\
& =4 \sum_{n=1}^{\infty}(-1)^{n-1} \frac{(4 n+1)\left(\begin{array}{c}
2 n \\
n
\end{array}\right)^{3}}{2^{6 n}} .
\end{aligned}
$$

This leads to:

$$
\frac{2}{\pi}=1-\sum_{n=1}^{\infty}(-1)^{n-1} \frac{(4 n+1)\left(\begin{array}{c}
2 n \\
n
\end{array}\right)^{3}}{2^{6 n}}=\sum_{n=0}^{\infty}(-1)^{n} \frac{(4 n+1)\left(\begin{array}{c}
2 n \\
n
\end{array}\right)^{3}}{2^{6 n}} .
$$

Other consequences of Theorem 5 are:

\section{Corollary 4}

$$
\begin{aligned}
& \frac{3 \sqrt{3}}{2 \pi}=\sum_{n=0}^{\infty}(-1)^{n}(6 n+1) \frac{\left(\frac{1}{3}\right)_{n}^{3}}{n !^{3}} \quad \frac{3}{\pi}=\sum_{n=0}^{\infty}(-1)^{n}(12 n+1) \frac{\left(\frac{1}{6}\right)_{n}^{3}}{n !^{3}} \\
& \frac{2 \sqrt{2}}{\pi}=\sum_{n=0}^{\infty}(-1)^{n}(8 n+1) \frac{\left(\frac{1}{4}\right)_{n}^{3}}{n !^{3}} \quad \frac{4 \sqrt{2-\sqrt{2}}}{\pi}=\sum_{n=0}^{\infty}(-1)^{n}(16 n+1) \frac{\left(\frac{1}{8}\right)_{n}^{3}}{n !^{3}} \\
& \frac{5 \sqrt{5-\sqrt{5}}}{2 \sqrt{2} \pi}=\sum_{n=0}^{\infty}(-1)^{n}(10 n+1) \frac{\left(\frac{1}{5}\right)_{n}^{3}}{n !^{3}} \quad \frac{5(\sqrt{5}-1)}{2 \pi}=\sum_{n=0}^{\infty}(-1)^{n}(20 n+1) \frac{\left(\frac{1}{10}\right)_{n}^{3}}{n !^{3}}
\end{aligned}
$$

Proof These series follow from the Wallis-type products for $\pi$ which can be found in [1] (see also [13], formulas (10), (11) and (19)):

$$
\frac{\sin (\pi m / k)}{\pi m / k}=\prod_{n=0}^{\infty} \frac{n k+k-m}{n k+k} \frac{n k+k+m}{n k+k}
$$

for $m, k$ positive integers with $m<k$. The choice $m=1, k=2$ leads to Wallis's product. If we take $m=1, k=3$, the product takes this form:

$$
\frac{3 \sqrt{3}}{2 \pi}=\frac{2 \cdot 4}{3 \cdot 3} \cdot \frac{5 \cdot 7}{6 \cdot 6} \cdot \frac{8 \cdot 10}{9 \cdot 9} \cdots
$$

As we did in the introduction with Wallis's product, we can rewrite this product as a series:

$$
\begin{aligned}
\frac{3 \sqrt{3}}{2 \pi} & =1-\frac{1}{3^{2}}-\frac{2 \cdot 4}{3^{2} \cdot 6^{2}}-\frac{2 \cdot 4 \cdot 5 \cdot 7}{3^{2} \cdot 6^{2} \cdot 9^{2}}-\cdots \\
& =1-\frac{1}{3^{2}} \sum_{n=0}^{\infty} \frac{\left(\frac{2}{3}\right)_{n}\left(\frac{4}{3}\right)_{n}}{(n+1) !^{2}} \\
& =1-\frac{1}{3^{2}} v\left(\frac{2}{3}, \frac{4}{3}\right)
\end{aligned}
$$


Using Theorem 5, we get the first formula. The second formula is found by taking $m=1, k=4$, the third one by taking $m=1, k=5$, and so on.

\section{Concluding remarks.}

1. The method used above to convert a product to a series can be applied directly to Euler's product formula for the sine-function:

$$
\sin \pi x=\pi x \prod_{m=1}^{\infty}\left(1-\frac{x^{2}}{m^{2}}\right) .
$$

The result is the following series:

$$
\frac{\sin \pi x}{\pi x}=\sum_{n=0}^{\infty} \frac{(-x)_{n}(x)_{n}}{n !^{2}} .
$$

which converges (by Raabe's test) for all $x \neq 0$.

2. Applying Theorem 5 to (16) results in this series:

$$
\frac{\sin \pi x}{\pi x}=\sum_{n=0}^{\infty}(-1)^{n} \frac{2 n+x}{x} \frac{(x)_{n}^{3}}{n !^{3}} .
$$

It can be found in Dougall's paper [4, p. 124 formula (16)]. All series in Corollary 4 are special cases of this general formula.

\section{References}

1. I. Ben-Ari, D. Hay, A. Roitershtein, On Wallis-type products and Pólya's urn schemes, Amer. Math. Monthly, 121, 5, 422-432 (2014).

2. N. D. Baruah, B. C. Berndt and H. H. Chan, Ramanujan's series for $1 / \pi$ : A Survey, Amer. Math. Monthly, 116, 7, 567-587 (2009).

3. W. Chu, Dougall's bilateral ${ }_{2} H_{2}$-series and Ramanujan-like $\pi$-formulae, Math. Comp. 80, 2223-2251 (2011).

4. J. Dougall, On Vandermonde's theorem and some more general expansions, Proc. Edinburgh Math. Soc., 25, 114-132 (1907).

5. A. R. Forsyth, A Series for $\frac{1}{\pi}$, Messenger of Mathematics, XII, 142-143 (1883).

6. J. W. L. Glaisher, On series for $\frac{1}{\pi}$ and $\frac{1}{\pi^{2}}$, Quarterly Journal of Pure and Applied Mathematics, XXXVII, 173-198 (1905-06).

7. J. Guillera, Series de Ramanujan: Generalizaciones y conjeturas. Ph.D. Thesis, University of Zaragoza, Spain (2007).

8. J. Guillera, Accelerating Dougall's ${ }_{5} F_{4}$ sum and the WZ-algoritm https://arxiv.org/ pdf/1611.04385.pdf (2016). Accessed 14 March 2017.

9. K. Knopp, Theory and Application of Infinite Series, Blackie, London, 2nd English ed. 4th reprint (1954).

10. P. Levrie, Using Fourier-Legendre expansions to derive series for $1 / \pi$ and $1 / \pi^{2}$, Ramanujan J. 22, no. 2, 221-230 (2010).

11. Z.-G. Liu, A summation formula and Ramanujan type series, J. Math. Anal. Appl. 389 (2), 1059-1065 (2012).

12. Z.-G. Liu, Gauss summation and Ramanujan-type series for $1 / \pi$, Int. J. Number Theory 8 (2), 289-297 (2012).

13. A. S. Nimbran, Generalized Wallis-Euler Products and New Infinite Products for $\pi$, Mathematics Student, Vol. 83, Nos. 1-4, 155-64 (2014). 
14. A. S. Nimbran, Deriving Forsyth-Glaisher type series for $\frac{1}{\pi}$ and Catalan's constant by an elementary method, Mathematics Student, Vol. 84, Nos. 1-2, 69-86 (2015).

15. Hessami Pilehrood Kh. and Hessami Pilehrood T., Generating function identities for $\zeta(2 n+2), \zeta(2 n+3)$ via the WZ-method, Electron. J. Combinatorics 15, \#R35 (2008).

16. S. Ramanujan, Modular equations and approximations to $\frac{1}{2}$, Quarterly Journal of Pure and Applied Mathematics, XLV, 350-372 (1914). Available at http://ramanujan. sirinudi.org/Volumes/published/ram06.pdf 